\title{
Classificação das áreas úmidas (AUs) hipersalinas da bacia hidrográfica do Rio Piancó-Piranhas-Açu e da Faixa Litorânea Norte de Escoamento Difuso.
}

\author{
Classification of wetlands (AUs) hypersaline the basin of River Piancó-Piranhas-Açu and \\ Coastal Range North Diffuse flow.
}

Silva, D. E. M. ${ }^{1}$; Costa, D. F. S. ${ }^{1}$

diegoemanoel22@gmail.com

\begin{abstract}
Resumo
A paisagem é resultado da interação entre fatores biofísicos e sociais, e se encontra em constante evolução. As alterações em um desses fatores implicam em ajustes nos demais, que compõem o sistema ambiental. Desse modo, o presente trabalho propõe uma análise das transformações ocorridas na paisagem do município costeiro de Brejo Grande/SE, com ênfase no uso e ocupação das terras. Para alcançar o referido objetivo os estudos foram norteados pela análise integrada, tendo como procedimentos metodológicos - pesquisa bibliográfica, análise de documentos cartográficos, trabalhos de campo e elaboração de mapas com técnicas de geoprocessamento. A análise efetuada confirma que as transformações na paisagem da área são consequências da atuação dinâmica de processos costeiros e fluviais, influenciados por ações antrópicas associadas às atividades produtivas, principalmente, a rizicultura, a carcinicultura e a cocoicultura.
\end{abstract}

Palavras-chave: dinâmica da paisagem, uso e ocupação das terras, atividades produtivas

\begin{abstract}
The landscape is the result of interaction between biophysical and social factors, and is constantly evolving. Changes in one of these factors imply adjustments in others, that make up the environmental system. Thus, this paper proposes an analysis of the transformations in the landscape of the coastal municipality of Brejo Grande / SE, with emphasis on the use and occupation of land. To achieve that objective studies were guided by integrated analysis, with the methodological procedures bibliographical research, analysis of cartographic documents, field work and preparation of maps with GIS techniques. The analysis performed confirms that the changes in the landscape of the area are the dynamic performance consequences of coastal and fluvial processes, influenced by human activities associated with productive activities, mainly to rice cultivation, shrimp farming and cocoicultura.
\end{abstract}

Keywords: landscape dynamics, use and occupation of land, productive activities

\section{INTRODUÇÃO}

Áreas úmidas (AUs) são ambientes inundados frequentemente, como rios, lagoas, lagos, várzeas, pântanos, planícies de inundação costeira, laguna, manguezais ou outras áreas marinhas alagáveis com menos de seis metros de profundidade em maré baixa, áreas naturais ou artificias, permanente ou temporária, de água corrente ou parada, doce, salobra ou salgada, onde são classificadas ao todo 42 tipos diferentes desses ecossistemas (JUNK; BAYLEY; SPARKS, 1989; BARBIER; ACREMAN; KNOWLER, 1997; RAMSAR, 2006; JUNK et al., 2104).

Logo, as áreas úmidas são considerados ecossistemas de transição entre os ambientes terrestre e marinho, sendo classificados entre AUs com nível de água estável e AUs com nível de 
água flutuante. No território brasileiro $90 \%$ das suas áreas úmidas são afetadas pelo sistema de pulso de inundação (AUs com nível de água flutuante), em que, esses ecossistemas somente têm seus estudos avançados no âmbito cientifico por Junk, Bayley, Sparks (op. cit.) (JUNK et al., 2015).

No ano de 1971 foi realizada a primeira convenção sobre a conservação e o uso sustentável das áreas úmidas na cidade de Ramsar no Irã, conhecida como Convenção de RAMSAR, gerando um tratado em que as áreas úmidas foram classificadas em cinco categorias: marinhas, estuarinas, lacustres, fluviais e pantanosas (RAMSAR, 2006). Todavia, apenas no ano 1993, o Brasil tornou-se signatário do tratado de RAMSAR publicado no decreto da $\mathrm{n}^{\circ} 1.905$, de 16 de maio de 1996, para a conservação das áreas úmidas no país (BRASIL, 1996; JUNK; PIEDADE, 2015).

De acordo com a classificação da convenção de RAMSAR (op. cit.), as AUs são distribuídas em três grupos: marinhas e costeiras (marinhas, estuarinas, lacustres/palustres), interiores (lóticas, lacustres, palustres, geotermais) e antrópicas (aquicultura, agricultura, mineração de sais minerais, urbana e industrial), nessa concepção as áreas litorâneas são distribuídas tanto no grupo marinho e costeiros, e no grupo dos antrópicos com a produção de sais minerais.

Vale salientar que esses ecossistemas são compreendidos por variáveis classificações em todo mundo (DIAS, 2014). No Brasil pode-se definir áreas úmidas de acordo com a Lei $\mathrm{n}^{\circ} 12.651$ de 25 de maio de 2012, que as define como superfície terrestre alagável periodicamente por água, podendo conter vegetação adaptada aos pulsos de inundação, sejam o fluído de característica doce, salobra ou salgada (BRASIL, 2012).

Prontamente, Diegues (1994) foi pioneiro na classificação das áreas úmidas no Brasil, com a produção do Inventário das Áreas Úmidas Brasileira, onde, foi abordada a importância desses ecossistemas de acordo com seus valores ecológico e sócio econômico. Nesse registro, encontra-se o pantanal mato-grossense, os ecossistemas costeiros (manguezais, lagoas, lagunas, salinas), várzeas de rios, lagoas continentais, igarapés (região amazônica) além de ecossistemas artificias (barragens, açudes e reservatórios).

Todavia, o primeiro estudo completo sobre as AUs no território brasileiro, foi a “Classificação dos Principais Tipos de AUs Brasileiras", onde foi levado em conta os fatores da dinâmica hidrológica, os parâmetros físicos e químicos e o padrão da estrutura botânica, de cada tipo de ecossistema caracterizado (JUNK et al., 2015). Classificação semelhante foi realizada por Junk no ano de 2013, sendo classificado as “Áreas Úmidas de América do Sul tropical” (JUNK, 2013 apud JUNK op. cit.).

No litoral semiárido brasileiro se encontra em maior parte nos estados do Rio Grande do Norte e Ceará, com uma extensão de aproximadamente $244 \mathrm{~km}$, onde localiza-se a porção final bacia hidrográfica do Rio Piancó-Piranhas-Açu (SILVA; GUEDES; COSTA, 2014) Prontamente, 
tem-se nessa região uma rica diversidade de AUs costeiras e estuarinas, naturais e artificiais, classificados ao grau de salinidade hipersalina, que proporcionam serviços ecossistêmicos de caráter essencial a população ribeirinha para suas manutenções na região, como, os principais de disponibilidade de alimentos, purificação da água potável, controle de inundação nas regiões costeiras, além de serviços recreativos e religioso cultural (MMA, 2010).

Todavia, as AUs hipersalinas representam ecossistemas fundamentais para o equilíbrio e manutenção da biodiversidade, isso devido suas localizações geográfica em ambientes planos que são afetados diretamente pelos pulsos de maré. Em regiões de climas áridos e semiáridos, devido as inundações pelos sistemas de marés, ocorrem deposições evaporíticas seguido das precipitações de sais conhecidos como, carbonatos, halitas e/ou sulfatos (SOARES; DOMINGUEZ, 2012; ARAÚJO et al., 2013). Tem-se as principias áreas úmidas tropicais hipersalinas costeiras: estuários, lagoas, lagunas, planícies hipersalinas, zona de mangues e apicuns (BURGER, 2000).

Assim o referido trabalho tem como objetivo principal realizar uma classificação das áreas úmidas costeiras localizada no litoral Semiárido Potiguar, mais precisamente no trecho da bacia hidrográfica do Rio Piancó-Piranhas-Açu e na Faixa Litorânea Norte de Escoamento Difuso, tendo como base a "Classificação dos Principais Tipos de AUs Brasileiras", classificação essa proposta por Junk et al. (2014).

\section{METODOLOGIA}

\section{1 Área de estudo}

O referente trabalho foi desenvolvido em áreas úmidas costeiras localizadas na porção litorânea da bacia hidrográfica do Rio Piancó-Piranhas-Açu e na Faixa Litorânea Norte de Escoamento Difuso, desde o município de Macau em sua porção leste, até o município de Areia Branca na porção oeste do litoral (Fig. 01). Em que as formações estuarinas e litorâneas encontradas na área de estudo são pertencentes à costa norte do Quaternário (KNOPPERS; KJERFVE, 1999). O clima predominante é o tropical quente e seco (semiárido), sua vegetação é compreendida pela savana estépica (Caatinga), de porte arbustivo/herbáceo, sua geomorfologia está contido de

planícies e tabuleiros costeiros, campos de dunas (barcanas e barcanóides), praias arenosas e lamosas além de falésias ativas (NIMER, 1989).

\subsection{Procedimentos metodológicos}


Os procedimentos metodológicos foram divididos em seis etapas: 1) um levantamento bibliográfico e cartográfico prévio da área estudada; 2) a montagem de uma base cartográfica digital; 3) a realização de um processamento digital das imagens; 4) classificar as AUs hipersalinas; 5) quantificar as áreas úmidas encontrada na área de estudo.

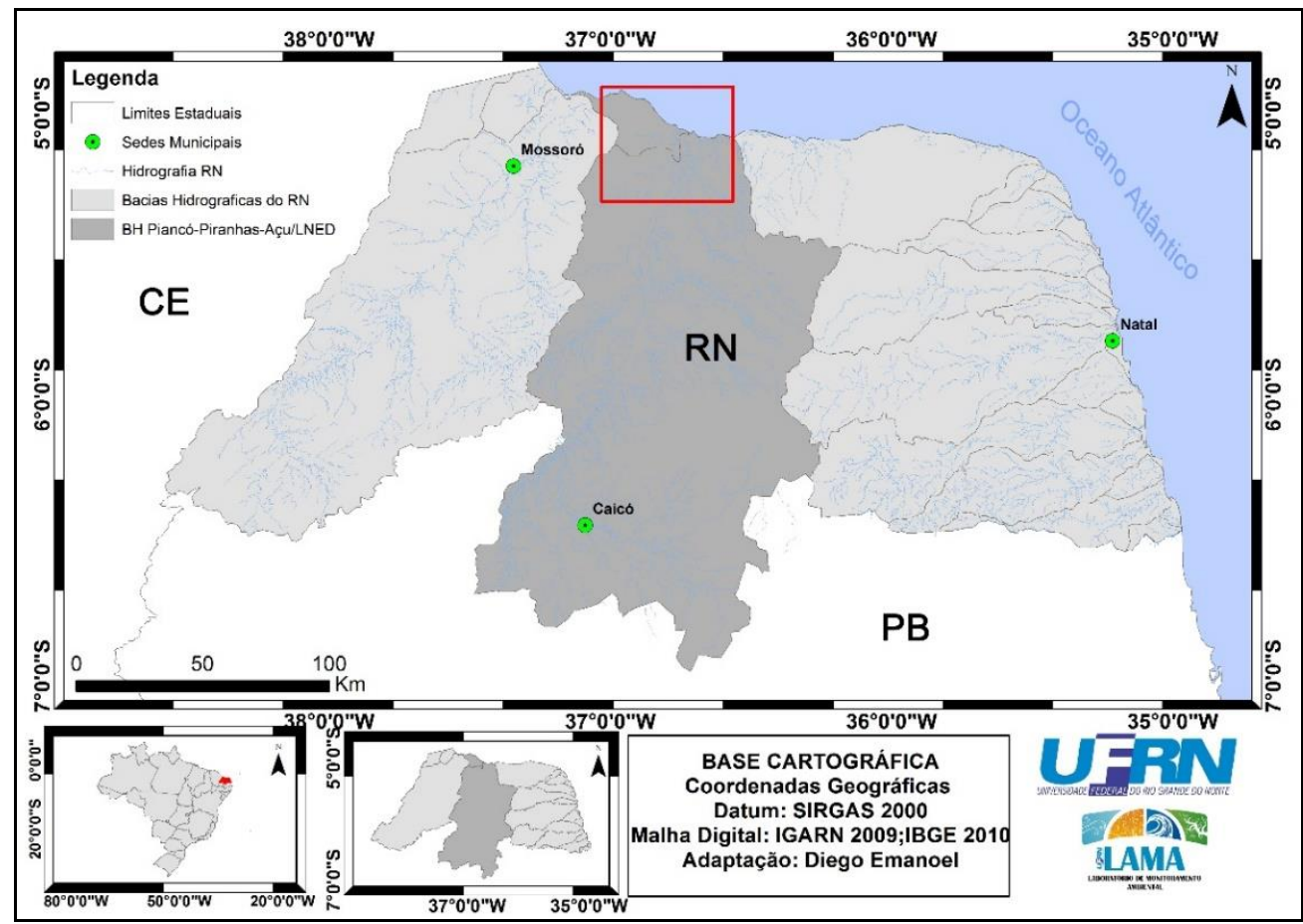

Figura 01. Mapa de localização da porção litorânea da bacia hidrográfica do Rio Piancó-Piranhas-Açu e na Faixa Litorânea Norte de Escoamento Difuso.

Fonte: Acervo dos autores.

O levantamento bibliográfico foi realizado a partir de pesquisas, em artigos científicos, de classificação entre A1 e B2 de acordo com o "qualis capes", além de trabalhos acadêmicos e livros que fundamentaram a pesquisa sobre a temática abordada, seguindo de uma revisão em acervos cartográficos disponibilizados por instituições federais e estaduais.

A montagem da base cartográfica digital foi realizada em ambiente de Sistema de Informação Geográfica (SIG), no primeiro momento constituiu na formação de um acervo de dados matriciais (raster), seguido no armazenamento de dados vetoriais (vetor) no formato shapefile que foram elaborados durante a identificação das áreas úmidas costeiras, e dados quantitativos (planilhas, gráficos, tabelas) na distribuição da variedade de ecossistemas identificados.

Para o processamento digital foram utilizadas imagens obtidas pelos satélites CEBERS 2B, foram utilizadas quatro imagens capturadas através do sensor HRC (High Resolution Camera) com resolução espectral de 2,7m, (órbita 148 C, ponto 106 - 1, de 23/02/2008; órbita 148 B, ponto 106 1, de 10/05/2009; órbita 148 B, ponto 106 - 1 e 2, de 29/07/2009) e uma imagem do sensor CCD 
(High Resolution CCD Camera) com resolução de 20m, (órbita 148 D, ponto 106, de 23/02/2008), todas as representações utilizadas são disponibilizadas gratuitamente pelo Instituto Nacional de Pesquisas Espaciais (INPE).

A próxima etapa do processamento digital foi realizada no software ENVI 4.7 (Versão Acadêmica), foi associada às bandas 3 na cor vermelha $(\mathrm{R})$, a banda 2 na cor verde $(\mathrm{G})$ e a banda 1 cor azul (B), onde foi produzido uma composição colorida em falsa cor representada por 321 (RGB) da imagem do sensor CCD. Logo em seguida foi realizado um georreferenciamento das imagens, onde foi utilizado o software ArcGIS 10.3 (Versão Acadêmica). O georreferenciamento foi feito na grade de coordenadas UTM (Universal Transversa de Mercartor) e pelas cartas topográficas (formato digital) da Superintendência do Desenvolvimento do Nordeste (SUDENE) (SB-24-X-B-IV Areia Branca e SB-24-X-B-V Macau), em escala de 1:200.000, a partir do SIRGAS 2000.

Após o georreferenciamento das imagens, as cenas do sensor HRC foram mosaicadas em ambientes de SIG, para o mapeamento das AUs antrópicas e hídricas, já para o mapeamento das AUs com presença de vegetação foi realizada uma fusão das imagens mosaicadas (Sensor HRC) com a imagem do sensor CCD, bem como todo o processo de manipulação e integração dos dados espaciais. Em seguida, procedeu com a delimitação das AUs através de vetorização manual, onde toda a elaboração e layout final dos mapas temáticos foram realizados com o auxílio do software ArcGIS v. 10.3 (acadêmica) $\left(\mathrm{ESRI}^{\odot}\right)$.

O processo seguinte em relação ao processamento digital foi analisar e classificar as AUs de acordo com a nova classificação das AUs (Áreas Úmidas) brasileira proposta também por Junk et al. (2014). Em seguida após o processo de identificação e classificação das áreas úmidas, os seguintes dados das AUs encontradas na área de estudo foram tabulados e quantificados, seguido da geração de gráficos e tabelas com os resultados obtido na classificação, todo o processo realizado com o auxílio do software Microsoft Excel $2016^{\circledR}$.

\section{RESULTADOS E DISCUSSÃO}

A partir do procedimento digital do referido trabalho, pode-se constatar que a porção litorânea da bacia hidrográfica do Rio Piancó-Piranhas-Açu e a Faixa Litorânea Norte de Escoamento Difuso foi possível identificar e mapear oito tipos de AUs hipersalinas, entres elas: estuários, lagoas e lagunas (Hidrografia); salinas solares e tanques de carcinicultura (Antrópicos e Hidrografia); Manguezais e Apicuns (Vegetação), criando um mosaico de ecossistemas costeiros identificados (Fig. 02). 


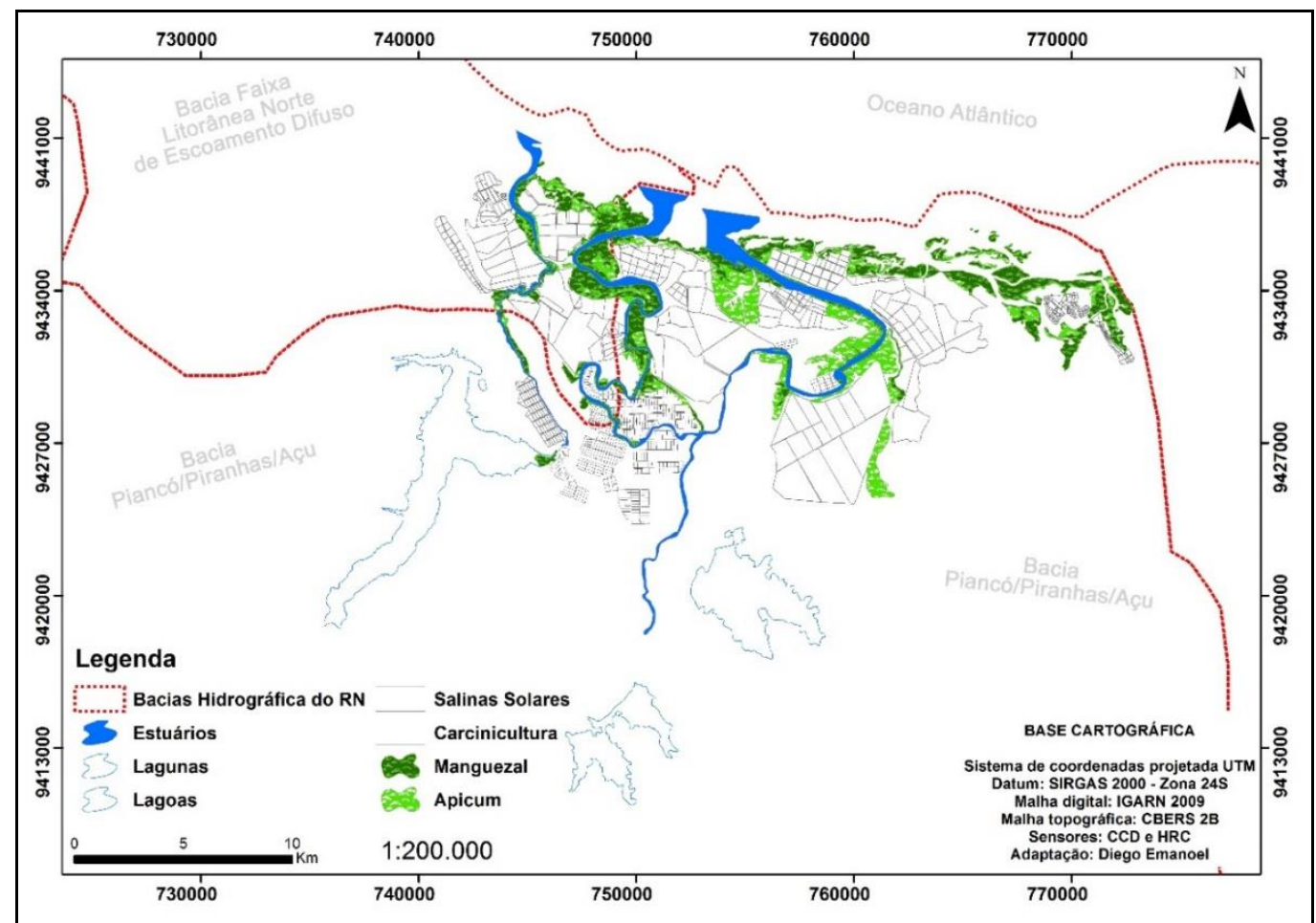

Figura 02. Mosaico de AUs hipersalinas da Bacia Hidrográfica do Rio Piancó-Piranhas-Açu e da Faixa Litorânea Norte de Escoamento Difuso.

Fonte: Acervo dos autores.

A classificação dos ecossistemas hipersalinos foi realizada logo após o trabalho cartográfico, levou-se como referência a classificação das AUs brasileiras proposta por Junk et al. (2014), ao seguir a metodologia proposta no trabalho referente, pode-se dividir o referido resultado em: sistemas, subsistemas, subclasses e macrohabitat. Os sistemas foram rotulados em AUs Costeiras e AUs antropogênicas, já ao se referir aos subsistemas apenas as AUs costeiras foram interpretadas, pois os ecossistemas antropogênicos apenas são qualificados de acordo com os macrohabitats, assim os subsistemas analisados foram as AUs sujeita aos impactos dos pulsos previsíveis de curta duração de maré, as separadas do mar com nível de água relativamente estáveis e variável, as subclasses levo o mesmo critério da classificação anterior, onde a única subclasse selecionada foi a AUs marinhas, logo, os macrohabitats foram todas os ecossistemas hipersalinos identificados no mapeamento (Qua. 01). 
Quadro 01. Classificação das AUs hipersalinas da Bacia Hidrográfica do Rio Piancó-Piranhas-Açu e da Faixa Litorânea Norte de Escoamento Difuso.

Fonte: Acervo dos autores.

\begin{tabular}{|c|c|c|c|c|}
\hline & SISTEMAS & SUBSISTEMAS & SUBCLASSES & MACROHABITAT \\
\hline \multirow{3}{*}{$\begin{array}{l}\text { ssim } \\
\text { de } \\
\text { acord }\end{array}$} & \multirow{5}{*}{ AUs costeiras } & \multirow{3}{*}{$\begin{array}{l}\text { AUs sujeita aos impactos dos pulsos } \\
\text { previsíveis de curta duração de maré }\end{array}$} & \multirow{5}{*}{ AUs marinhas } & $\begin{array}{l}\text { Manguezais nas } \\
\text { embocaduras de rios } \\
\text { (zonas estuarinas) }\end{array}$ \\
\hline & & & & $\begin{array}{l}\text { Áreas Hipersalinas } \\
\text { (Apicum) }\end{array}$ \\
\hline & & & & Estuários \\
\hline \multirow{2}{*}{$\begin{array}{l}\mathrm{o} \\
\mathrm{com}\end{array}$} & & $\begin{array}{l}\text { AUs separadas do mar com nível de } \\
\text { água relativamente estáveis }\end{array}$ & & Laguna \\
\hline & & $\begin{array}{l}\text { AUs separadas do mar com nível de } \\
\text { água variável }\end{array}$ & & Lagoas \\
\hline \multirow{2}{*}{$\begin{array}{l}\text { o } \\
\text { quad }\end{array}$} & \multirow{2}{*}{$\begin{array}{c}\text { AUs } \\
\text { antropogênicas }\end{array}$} & - & - & Salinas Solares \\
\hline & & - & - & Carcinicultura \\
\hline
\end{tabular}

ro acima, pode-se classificar os ecossistemas em AUs costeiras divididas em três subsistemas, AUs sujeita aos impactos dos pulsos previsíveis de curta duração de maré onde esses são introduzidos os macrohabitats de manguezais, apicuns e estuários, o estuário sendo o único ecossistema totalmente aquático nesse subsistema, o segundo subsistema foi AUs separadas do mar com nível de água relativamente estáveis, que abarcou apenas o sistema lagunas e o subsistema das AUs separadas do mar com nível de água variável incluindo apenas as lagoas costeira, todos esses ecossistemas são introduzidos na subclasse AUs marinhas.

Logo, o outro sistema presente na classificação foi o das AUs antropogênicas, os ecossistemas introduzidos nesse sistema apenas consistem em macrohabitats, as salinas solares e os tanques de carcinicultura, mesmo sendo localizados em áreas marinhas não são definidos em subsistema e subclasses devido a atuação antrópica na construção desses ecossistemas. Prontamente, Diegues (1994) realizou estudos semelhante classificando a referida área de estudo como Zona Salina do Rio Grande do Norte, devido as salinas solares frequente na região.

De acordo com a classificação das AUs, foi totalizado a extensão ocupada pelos ecossistemas na área de estudo, nos dados quantificados não foram separados por sistemas e sim uma ocupação geral das AUs em unidade de medida hectare (ha) (Fig. 03) 


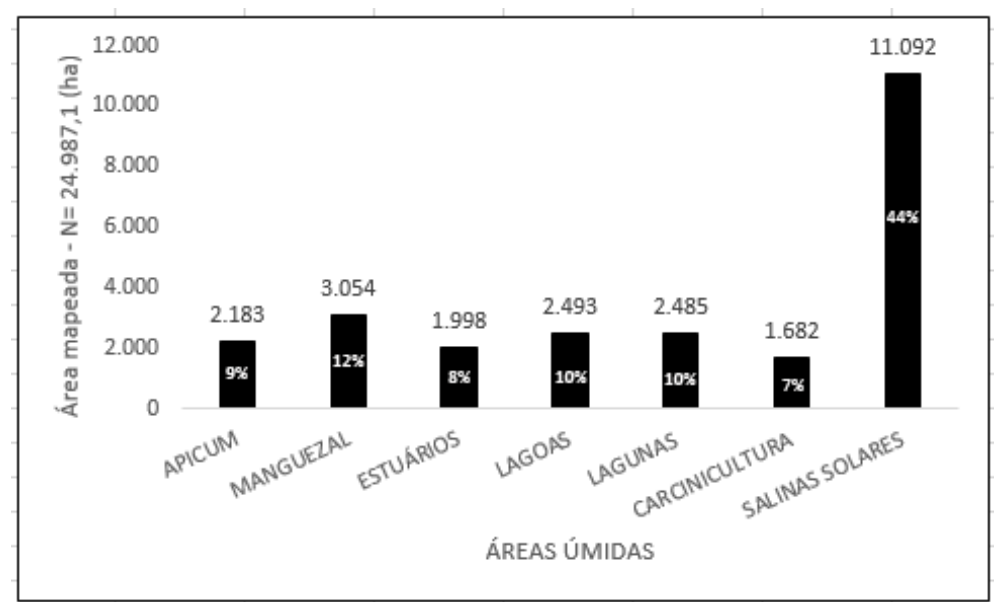

Figura 03. Histograma de ocupação das AUs na área da Bacia Hidrográfica do Rio Piancó-Piranhas-Açu e da Faixa Litorânea Norte de Escoamento Difuso.

Fonte: Acervo dos autores.

Todavia, foram mapeados 24.987,1 ha de AUs hipersalinas na área de estudo, o ecossistema mais maior presença de ocupação da terra, foi as salinas solares com cerca de 11.092ha cerca de $44 \%$ de todo o total mapeado, os outros seis ecossistemas mapeados obtiveram um padrão homogêneo de ocupação, dividindo-se a em sistema de classificação, tem-se que as áreas catalogadas em AUs antropogênicas totalizando dois habitats, somam juntos mais da metade da área ocupada, mais precisamente 12.774 ha equivalente a $51 \%$ do total, já no que corresponde aos habitats de AUs costeiras (apicum, manguezal, estuários, lagoas, lagunas) totalizando cerca de 12.213 ha menos da metade do total, equivalente a $49 \%$ do total. Ao analisar os dados anteriores pode distinguir a área mapeada praticamente dividida em uma metade AUs antropogênicas e a outra em AUs costeiras.

Desde modo, a maior parte do território mapeado são ecossistema artificiais, ou seja, construído por ação antrópica, assim foi possível quantificar também a quantidade de tanques que os habitats salinas solares e carcinicultura preenchem na porção litoral (Fig. 04). Vale salientar que os dados obtidos são da quantidade de tanques mapeados, sendo eles de diversas formas e tamanhos. 


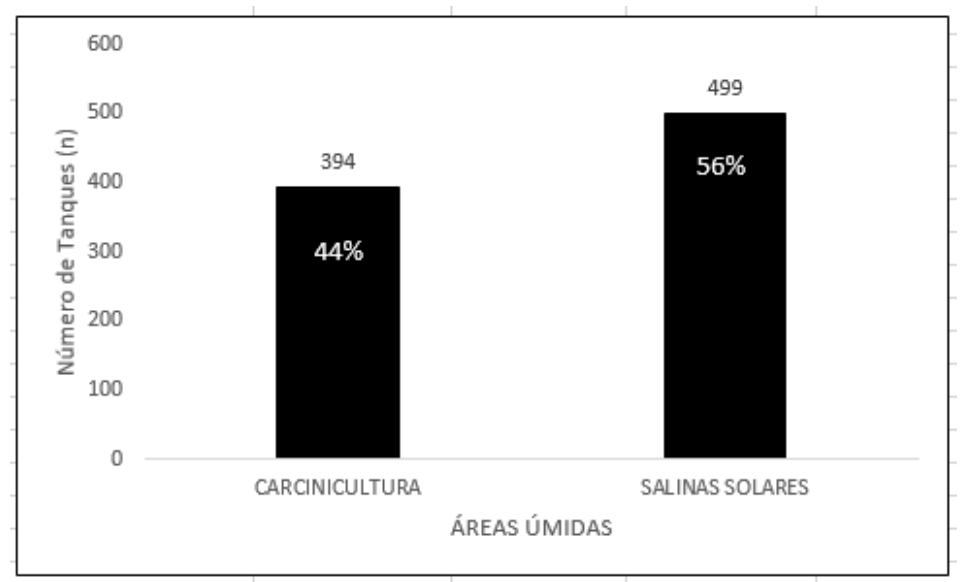

Figura 04. Histograma da quantidade de tanques se encontra nas AUs antropogênicas. Fonte: Acervo dos autores.

Assim, as salinas solares além de suportarem a maior ocupação do solo na área de estudo, também deleitar-se da maior quantidade de tanques alagáveis, com quase 500 tanques mapeados, cerca de $56 \%$ do total identificado, já os tanques de carcinicultura são o que ocupam o menos espaço na área de estudo, obtendo uma diferença de quase 100 tanques em relação as salinas solares, ocupando um total de $44 \%$ de todos os tanques cartografados.

\section{CONSIDERAÇÕES FINAIS}

A parti do mapeamento obtido através da metodologia de mosaicagem e fusão das imagens CBERS 2B, em que para as condições locais, a classificação visual gerou resultados positivos para a classificação das AUs hipersalinas, além do fator do conhecimento prévio da área, que se tornou imprescindível para a qualidade do resultado da classificação visual/manual, onde para confirmar o ponto positivo no trabalho, exalta-se o valor total mapeado $(24.987,1$ ha).

Sequencialmente, tem-se que o método escolhido para a classificação se encontra de forma parcialmente positiva, onde pode-se favorecer o esquema utilizado por Junk et al. (2014) com uma divisão coesa entre vários tipos de classe, já a questão que não corresponda satisfatoriamente com a classificação seja, a ausência da introdução dos subsistemas e subclasses ao se referir as AUs antropogênicas, além de na classificação brasileira não conter as salinas solares nesse sistema, onde o mesmo foi introduzido na interpretação dos autores deste trabalho, muito deve-se pelo fato de ser uma classificação em escala nacional deixando a desejar em alguns critérios de conhecimento. 


\section{REFERÊNCIAS BIBLIOGRÁFICAS}

ARAÚJO, D. M.; MEDEIROS, D. H. M.; COSTA, D. F. S.; ROCHA, R. M. Geoprocessamento aplicado a caracterização ambiental da vegetação em zonas úmidas hipersalinas. In: XVI Simpósio Brasileiro de Sensoriamento Remoto - SBSR, 16., 2013, Foz do Iguaçu. Anais... São José dos Campo - SP: Instituto Nacional de Pesquisas Espaciais - INPE, 2011. p. 2889- 2896.

BARBIER, E. B.; ACREMAN, M.; KNOWLER D. Economic valuation of wetlands: A guide for policy makers and planners. Gland: Ramsar Convention Bureau, 1997. 116 p.

BRASIL. Decreto $\mathbf{n}^{\mathbf{0}} \mathbf{1 . 9 0 5}$, de 16 de maio de 1996. Promulga a Convenção sobre Zonas Úmidas de Importância Internacional, especialmente como Habitat de Aves Aquáticas, conhecida como Convenção de Ramsar, de 02 de fevereiro de 1971. Disponível em: <http://www.planalto.gov.br/ccivil_03/decreto/1996/D1905.htm>. Acesso em: 11 de abr. 2016.

BRASIL. Lei no 12.651 de 25 de maio de 2012. Diário Oficial da União. 2012.

BURGER, M. I. Situação e ações prioritárias para conservação de banhados e áreas úmidas da Zona Costeira. Brasília: ANP, 2000.

DIAS, A. P. Análise espacial aplicada à delimitação de áreas úmidas da planície de inundação do médio Araguaia. 2014. 91 f. Dissertação (Mestrado em Ciências Florestais e Ambientais) - Faculdade de Engenharia Florestal, Universidade Federal de Mato Grosso, Cuiabá, 2014.

DIEGUES, A. C. S. An inventory of Brazilian wetlands. Gland, Switzerland: IUCN - The World Conservation Union, 1994.

JUNK, W. J.; BAYLEY, P. B.; SPARKS, R. E. The flood pulse concept in river-floodplain systems. In: DODGE, D. P. (Org.). Proceedings of the International Large River Symposium. Ottawa: Canadian Special Publication of Fisheries and Aquatic Science, 1989. p. 110-127.

JUNK, W. J.; PIEDADE, M. T. F. Áreas Úmidas (AUs) Brasileiras: Avanços e Conquistas Recentes. Boletim ABLimno, v. 41, n. 2, p. 20-24, 2015.

JUNK, W. J.; PIEDADE, M. T. F.; LOURIVAL, R.; WITTMANN, F; KANDUS, P.; LACERDA, L. D.; BOZELLI, R. L.; ESTEVES, F. A.; NUNES DA CUNHA, C.; MALTCHIK, L.; SCHÖNGART, J.; SCHAEFFERNOVELLI, Y.; AGOSTINHO, A. A. Brazilian wetlands: their definition, delineation, and classification for research, sustainable management, and protection. Aquatic Conservation: Mar. Freshw. Ecosyst., v. 24, p. 5-22, 2014.

JUNK, W. J.; PIEDADE, M. T. F.; LOURIVAL, R.; WITTMANN, F; KANDUS, P.; LACERDA, L. D.; BOZELLI, R. L.; ESTEVES, F. A.; NUNES DA CUNHA, C.; MALTCHIK, L.; SCHÖNGART, J.; SCHAEFFERNOVELlI, Y.; AGOSTINHO, A. A.; NÓBREGA, R. L. B.; CAMARGO, E. Definição e Classificação das Áreas Úmidas (AUs) Brasileiras: Base Científica para uma Nova Política de Proteção e Manejo Sustentável. In: NUNES DA CUNHA, C.; PIEDADE, M. T. F.; JUNK, W. J. (Org.) Classificação e Delineamento das Áreas Úmidas Brasileiras e de seus Macrohabitats. Cuiabá: EdUFMT, 2015. p. 13-82.

KNOPPERS, B.; KJERFVE, B. Coastal Lagoons of Southeastern Brazil: physical and biogeochemical characteristics. In: PERILLO, G. M. E.; PICOLLO, M. C.; PINO-QUIVIRA, M. Estuaries of South America: Their Geomorphology and Dynamics. Heidelberg: Springer, 1999.

MINISTÉRIO DO MEIO AMBIENTE - MMA. Panorama da conservação dos ecossistemas costeiros e marinhos no Brasil. Brasília: MMA/SBF/GBA, 2010. 
NIMER, E. Climatologia do Brasil. Rio de janeiro: IBGE, Departamento de Recursos Naturais e Estudos Ambientais, 1989.

RAMSAR. The Ramsar Convention Manual: a Guide to the Convention on Wetlands (Ramsar, Iran, 1971), 4 ed. Gland, Switzerland: Ramsar Convention Secretariat, 2006.

SILVA, D. E. M.; GUEDES, D. R. C.; COSTA, D. F. S. Caracterização morfométrica de sistemas flúviolagunares no litoral semiárido do Brasil (RN). In: Encontro de Geografia do Rio Grande do Norte, $21^{\circ}, 2014$, Caicó. Anais... Caicó: XXI Encontro de Geografia do Rio Grande do Norte, 2014.

SOARES, G. S.; DOMINGUEZ, J. M. L. Zonas úmidas na planície costeira do rio Itapicuru, litoral norte do estado da Bahia, Brasil: classificação e controles ambientais. Revista da Gestão Costeira Integrada, v, 12, n. 2, p. 223-237, 2012.

\section{AGRADECIMENTOS}

A equipe agradece ao Laboratório de Monitoramento Ambiental (UFRN/CERES), pelo apoio nos trabalhos de campo e de gabinete, assim como a PROPESQ/UFRN (PVF10463-2014) e ao CNPq (MCTI/CNPQ/Universal Proc.447227/2014-9), pelo apoio financeiro. Os autores também agradecem aos avaliadores anônimos da revista, cujas contribuições foram valorosas para a publicação deste trabalho.

Recebido em: 14/08/2016

Aceito para publicação em: 01/10/2016 